\title{
CREENCIAS DE LOS ALUMNOS DEL GRADO DE EDUCACIÓN SOBRE EL PROCESO DE APRENDIZAJE DE LA LENGUA INGLESA
}

\author{
BELIEFS OF TRAINING SCHOOL STUDENTS ABOUT THE PROCESS OF \\ LEARNING ENGLISH
}

\author{
Raquel Marín Chamorro, Teresa Alzás García, Silvia Villares Hurtado. \\ Universidad de Extremadura
}

\author{
Correspondencia: Raquel Marín Chamorro \\ Correo: raquelmarinc@movistar.es
}

Recibido: 17/05/2018; Aceptado: 18/02/2019

DOI: 10.17398/0213-9529.39.1.71

\section{Resumen}

En la actualidad el aprendizaje del inglés está presente en todas las etapas educativas. Por ello, en este artículo se ha considerado oportuno analizar las creencias sobre el aprendizaje del inglés. Para ello, se ha contado con la colaboración de dos grupos de alumnos que cursan la asignatura de Inglés en el Grado en Educación Infantil y en el Grado en Educación Primaria de la Universidad de Extremadura. La elección de esta muestra tiene gran interés, considerando el objeto de investigación, dado que en el futuro serán docentes y trabajarán el inglés dentro del aula, posibilitando centros educativos bilingües de calidad. Los datos recogidos se han obtenido a través del cuestionario Beliefs about Language Learning Inventory ("Creencias sobre el aprendizaje de una lengua" basado en el cuestionario de Horwitz 1987, adaptado por Richards y Lockhart 1994) con el objetivo de conocer si existen diferencias significativas en las creencias del alumnado, a través de un análisis descriptivo e inferencial sobre sus preconcepciones según el sexo y especialidad que cursen.

Palabras claves: Creencias, proceso de aprendizaje, proceso de adquisición lenguas, Inglés.

\begin{abstract}
Nowadays, the process of learning English can be found at all stages of education. That is why, in this article, it has been considered appropriate to analyze the beliefs students have regarding this process. For this purpose we have counted on the collaboration of two groups of students, from the specialties of Early Education and Primary Education at Universidad de Extremadura. The choosing of this sample is not random, as these students will become teachers and educators who shall work on the English language within classrooms, enabling quality bilingual schools. All collected data has been obtained through a test grounded on Beliefs about Language Learning Inventory which is based on the Horwitz test from 1987,and was adapted by Richards and Lockhart in 1994. The goal of the test was to identify any meaningful differences among student beliefs and elaborate a descriptive and inferential analysis of their preconceptions according to sex and specialty..
\end{abstract}

Key Words: Beliefs, Language acquisition process, education process, English Language. 


\section{INTRODUCCIÓN}

La investigación sobre las creencias de los profesores se ha incrementado de manera notable en los últimos años. La publicación del International Handbook of Research on Teachers' Beliefs, coordinado por Fives y Gill (2015) puede indicar que la investigación sobre el pensamiento del profesorado se ha convertido actualmente en un tema relevante a nivel internacional. Las creencias del profesorado y sus sistemas de valores son importantes porque definen sus concepciones sobre la docencia y constituyen el marco de referencia en el que se van a diseñar las estrategias de enseñanza que van a utilizar en clase (Cheng, Chan, Tang y Cheng, 2009). Es importante profundizar en las creencias para erradicar las ideas erróneas que tiene el futuro profesorado sobre la lengua inglesa y para que aprendan a comunicarse mejor, ya que esta lengua se ha convertido en la llave que nos permite construir espacios de interacción y comunicación con otras personas (Marín, 1999).

En España el estudio de las lenguas extranjeras se ha minusvalorado debido a que los ciudadanos pertenecientes a países con una lengua más extendida y reconocida internacionalmente, tienen menos necesidad de hablar otra lengua (Martínez 2003). Otro factor que puede haber influido, es que en los países donde el doblaje de películas es una práctica habitual, obtienen peores resultados en el aprendizaje de otras lenguas que en los países donde el mundo audiovisual está subtitulado (Talaván, 2013).

Este artículo describe los resultados de un estudio cuantitativo para indagar las creencias de estudiantes en formación inicial en la enseñanza-aprendizaje del inglés en el Grado de Educación Infantil y Primaria de la Universidad de Extremadura en la Facultad de Ciencias de la Educación, y cómo estas creencias inciden en el aprendizaje de la lengua inglesa.

\section{Creencias.}

No hay un acuerdo general sobre lo que se entiende por creencia, pero sí sobre la importancia de su estudio. Las creencias que tenemos determinan con frecuencia nuestra forma de actuar e influyen en la toma de decisiones. Al mantener una creencia se adquiriere una disposición para actuar de determinada forma. Una definición del concepto de creencias, es la que propone Ramos (2007, p. 21):

Las creencias son ideas relativamente estables que tiene un individuo sobre un tema determinado, forjadas a través de su experiencia personal bajo la influencia de un proceso de construcción social, agrupadas en redes o sistemas, de cuya veracidad está convencido y que actúan como un filtro a través del cual percibe e interpreta el mundo que lo rodea, tomando sus decisiones de acuerdo con ello.

La importancia de las creencias se basa en que pueden ser conscientes o inconscientes, tienen un componente cognitivo evaluativo y afectivo, proporcionan una base para la acción y son resistentes al cambio (Borg, 2011, p. 370). Pajares (1992) sostiene que las creencias están basadas en evaluaciones y juicios, no requieren de pruebas de ningún tipo e influye mucho la toma de decisiones.

La concepción que nuestros alumnos y futuros maestros tienen sobre el proceso de aprendizaje está condicionada por la experiencia que han ido adquiriendo a lo largo de su etapa educativa en contextos educaciones formales y que según Asensio y Ruiz de Miguel (2015) plantea que son las creencias más difíciles de modificar. Estas creencias están basadas en el sentido común y surgen de la observación de situaciones de clase que ellos han vivido en 
sus prácticas de enseñanza y en su vida como estudiante; hacen lo que han visto hacer; creen lo que han visto que otros creen. Pajares (1992) plantea que el cambio de creencias es difícil, porque suelen formarse pronto y tienden a mantenerse incluso a pesar del tiempo, la escolarización o la experiencia.

De manera general, consideramos que el sistema de creencias que los alumnos tienen sobre su propio proceso de aprendizaje, influye en gran medida en los resultados que estos obtienen en las diferentes asignaturas

Según Espinosa (2014) partir de las ideas previas es un requisito para explorar y desafiar concepciones erróneas; es probable que las creencias perjudiciales e incompatibles con tendencias que se consideran en la actualidad válidas y coherentes incidan negativamente en la futura docencia del profesorado en formación y repercuta negativamente en el aprendizaje de su alumnado.

Es importante que, como docentes, uno de nuestros papeles fundamentales sea la escuchar activa sobre las opiniones, creencias e ideas de nuestros alumnos para poder así guiar nuestra intervención educativa hacia el camino que mejor se ajuste a sus necesidades.

De esta manera, si los alumnos tienen creencias erróneas hacia el aprendizaje de un determinado contenido o disciplina, estas convicciones podrían llevarles a tener mayor dificultad en el proceso de enseñanza-aprendizaje. Así, las creencias determinarán las acciones de los estudiantes y orientarán sus conductas. McCarthy (1991), establecía un ciclo sobre la relación entre las creencias y la acción (figura 1):

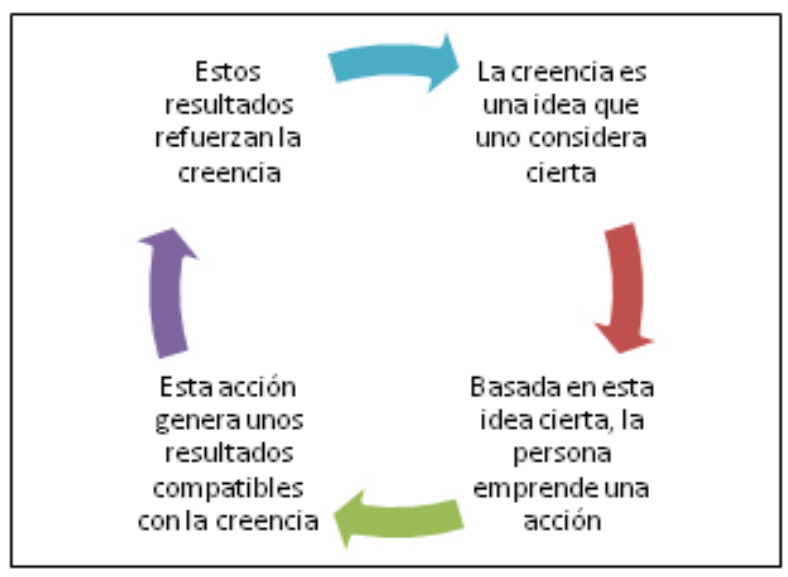

Figura 1. Ciclo de la relación creencias - acción de McCarthy (1991)

En este sentido, el contexto educativo, y en concreto el profesorado, tendrán gran influencia sobre las capacidades de su alumnado, pudiendo reforzar las creencias positivas y pudiendo minimizar las creencias erróneas. De esta manera, se ayuda a los alumnos a crear autopercepciones afirmativas adecuadas a sí mismo y adecuadas a su proceso de desarrollo y aprendizaje. Porque como decía Zimmerman 1990, "Ias ideas alternativas de los estudiantes se vuelven obstáculos en el aprendizaje, influyendo en sus resultados" (p. 10), de ahí que una de las funciones principales en los docentes sea combatir las creencias que puedan influir negativamente en el alumnado. 


\section{Creencias de los futuros docentes sobre el aprendizaje de un segundo idioma.}

Las creencias constituyen un elemento de gran relevancia en la formación de profesores de lenguas. Estas se refieren a todas aquellas proposiciones e ideas preconcebidas acerca de la forma y condiciones necesarias para el aprendizaje de una lengua extranjera. Estas frecuentemente se mantienen y son resistentes al cambio e influyen en el mayor o menor éxito con que se desarrollen sus procesos de aprendizaje. Los futuros docentes al entrar en el mundo de la enseñanza aportan sus teorías implícitas, su conocimiento personal, sus creencias, etc., acerca de lo que es la enseñanza y de cómo ha de ponerse en práctica.

Los estudios realizados manifiestan que los futuros docentes de una segunda lengua poseen un sistema inconsciente de creencias que, en ocasiones, difieren de los procedimientos sobre la enseñanza considerados apropiados (Barrios, 2006 y 2014). Estas creencias provienen de sus experiencias como alumnos (Pajares, 1992), de las observaciones que realizan durante las prácticas en los centros (Borg, 2003), de sus primeras experiencias docentes (Popko, 2005). En esta misma línea, Martínez (2005) considera que en la formación de creencias pueden influir también los factores de personalidad, la investigación y la metodología utilizada en las aulas.

Díaz y Morales (2015), piensan que Horwitz (1999) ha tenido mucha influencia y es pionera en las investigaciones sobre las creencias del aprendizaje de la lengua extranjera. Esta autora nos dice que todos tenemos creencias respecto al aprendizaje de una lengua extranjera, y usamos unas estrategias para conseguir el aprendizaje. Estas creencias se convierten en obstáculos del aprendizaje, si se han formado como resultado de experiencias negativas. Diseña un cuestionario que aborda las creencias de los estudiantes en relación al éxito y/o fracaso frente al aprendizaje del idioma; describe las creencias sobre la necesidad de conocer e involucrarse en la cultura de habla inglesa, así como las estrategias necesarias para adquirir el idioma; en otros ítems se exponen las creencias sobre la importancia y utilidad de aprender una lengua extranjera y afronta por último, las preguntas sobre creencias relacionadas con las reacciones de miedo y preocupación ante determinadas situaciones o personas. Murphy, Delli y Edwards (2004) creen que es al inicio del proceso educativo, cuando se puede intervenir en esas creencias y se puedan identificar las que puedan fomentar resultados positivos.

Northcote (2009) diseñó un estudio para indagar en las creencias sobre la enseñanza y aprendizaje de tres grupos de profesores y estudiantes que enseñaban y estudiaban en un programa de formación de profesores en una universidad australiana. Para recoger los datos utilizaron un cuestionario especialmente diseñado para este efecto, entrevistas y diarios de reflexión. Los resultados de la comparación entre las creencias de estudiantes y académicos acerca de la enseñanza y aprendizaje mostraron un alto grado de similitud en torno a cuatro grandes tópicos: creencias acerca de profesores y alumnos, creencias acerca de los procesos de enseñanza y aprendizaje, creencias acerca del contenido enseñado y aprendido, y creencias acerca de los propósitos de la enseñanza y el aprendizaje.

Finalmente, Barrios (2014) concluye que los formadores deberían partir de estas ideas y adaptar los contenidos de los programas de estudios para ayudar a los futuros maestros a reflexionar sobre la relación entre los nuevos conceptos y sus creencias preconcebidas.

Los procesos de desarrollo y formación docente, para que sean exitosos, deben considerar el sistema de creencias de esos profesores. No hacerlo llevará a que esas 
creencias, basadas en estilos de enseñanza-aprendizaje tradicionales, se conviertan en un impedimento para la implementación de nuevas estrategias o herramientas didácticas.

En esta línea, analizaremos las creencias de nuestros alumnos para poder trabajarlas con un enfoque constructivista y orientarlas hacia los aspectos positivos, y que puedan influir así efectivamente en el proceso de enseñanza-aprendizaje de la lengua extranjera.

\section{CONTEXTUALIZACIÓN.}

El trabajo de investigación que presentamos se centra en la asignatura de carácter obligatorio Inglés I, perteneciente a los Grados de Educación Infantil y Educación Primaria de la Universidad de Extremadura. Dicha asignatura tiene un diseño y un enfoque basados en el ESP (English for Specific Purposes) o Inglés para Fines Específicos cuyo objetivo es preparar al alumnado para impartir inglés a niños en la etapa de Infantil (3-5 años), o de 6 a 11, en el caso de Educación Primaria.

Inicialmente y dado que todos los alumnos de Grado tienen que cursar la asignatura y, por tanto, es un grupo muy numeroso y heterogéneo, se hace necesario identificar el nivel de competencia de sus integrantes y sus habilidades para el aprendizaje de esta lengua por lo que realizamos un estudio sobre su nivel de competencia comunicativa en cada una de las destrezas del que como resultado final obtenemos los datos mostrados en el siguiente gráfico (Figura 2):

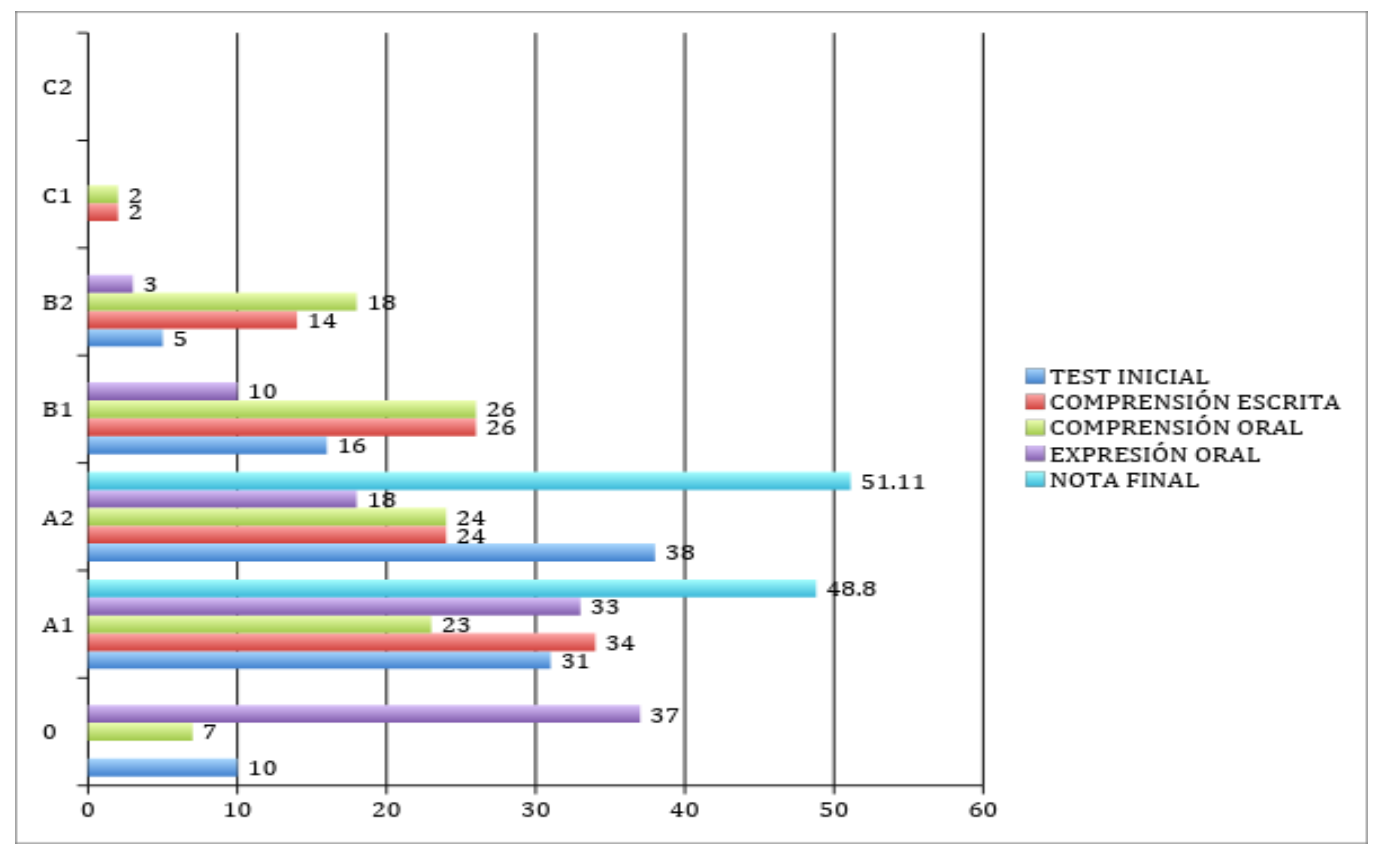

Figura 2. Nivel de competencia comunicativa

Podemos observar que la gran mayoría presenta dificultad en la expresión oral, con una competencia inexistente o preliminar, frente a los resultados obtenidos en las demás destrezas lingüísticas.

A lo largo de nuestra carrera docente, hemos detectado que sigue existiendo un problema en relación con la competencia comunicativa ya que, tras 10-12 años de estudio del 
inglés, muestran resultados muy bajos en el idioma destacando una gran dificultad para la destreza oral.

A partir de estas consideraciones se han establecido los siguientes objetivos de investigación:

- Analizar las creencias presentes en el aprendizaje del Inglés en estudiantes de Educación. Lo que requiere los siguientes objetivos específicos:

- Realizar un perfil académico del alumando que cursa la asignautura de Inglés.

- Describir las creencias presentes en el aprendizaje del Inglés según el sexo.

- Conocer las diferencias significativas relativas a las creencias presentes en el aprendizaje del Inglés según el sexo.

- Conocer las diferencias significativas relativas a las creencias presentes en el aprendizaje del Inglés según el Grado (Educación Infantil y Educación Primaria).

\section{METODOLOGÍA.}

Para establecer un diagnóstico inicial sobre la consideración del alumnado acerca de la asignatura de inglés, se ha elaborado un cuestionario basado en las concepciones que tiene el alumnado sobre la enseñanza de lenguas extranjeras, con el objetivo específico posterior de contrastar sus respuestas con el proceso de aprendizaje del inglés que han experimentado a lo largo de su vida, y poder así esclarecer creencias.

Además del cuestionario, se ha utilizado una escala Likert que recoge un conjunto de ítems sobre las creencias en relación con al aprendizaje del Inglés, en donde el alumnado tendrá que valorar el grado de acuerdo o desacuerdo con las afirmaciones presentadas. Concretamente es una herramienta que está basada en el cuestionario de Horwitz (1987), adaptado por Richards y Lockhart (1994) y además ha sido validada por expertos a quienes se les pidió que evaluasen la estructura, extensión, la adecuación del lenguaje y del cuestionario a la información que se pretende recabar, la relevancia de los ítems conforme a los objetivos, así como cualquier otra observación que quisieran formular.

Para el análisis conjunto de los datos cuantitativos obtenidos, a través de las citadas herramientas, se ha utilizado el método estadístico. Concretamente, el análisis de los datos se ha realizado con apoyo del programa estadístico SPSS (Statistical Package for the Social Sciences).

En cuanto a la población que ha colaborado en el cuestionario, está formada por futuros docentes que actualmente cursan el Grado de Educación Infantil y el Grado de Educación Primaria, que tendrán que impartir la asignatura de inglés a menores de entre 3 y 11 años de edad.

En concreto, la muestra está constituida por 226 estudiantes en la que el $94 \%$ de la muestra tiene entre 18 y 25 años, siendo el $20 \%$ hombres, de los cuales el $80 \%$ cursa estudios del Grado de Educación Primaria y el $20 \%$ el de Educación Infantil; y el $80 \%$ de la muestra son mujeres, de las cuales el 58,6 \% realizar el Grado de Educación Infantil y el 41,4 $\%$ el de Educación Primaria. (Tabla 1). 
Tabla 1. Distribución de frecuencias según Sexo y Grado

\begin{tabular}{lcccc}
\hline & \multicolumn{2}{c}{ Hombre } & \multicolumn{2}{c}{ Mujer } \\
\hline Grado que cursas actualmente & Frecuencia & Porcentaje válido & Frecuencia & Porcentaje válido \\
\hline Grado en Educación Infantil & 9 & 20,0 & 106 & 58,6 \\
Grado en Educación Primaria & 36 & 80,0 & 75 & 41,4 \\
\hline Total & 45 & 100,0 & 181 & 100,0 \\
\hline
\end{tabular}

Perfil académico del alumnado que cursa la asignatura de Inglés.

Conocida la muestra, pasamos a analizar ciertos datos académicos del conjunto de estudiantes que pueden tener cierta relevancia en cuanto al estudio del inglés en el Grado de Educación de Infantil y Primaria de la Facultad de Educación (Badajoz), que nos permite conocer el perfil de académico del alumnado.

En este sentido, cabe señalar que en el caso de los alumnos el $64,4 \%$ ha cursado en Bachillerato la especialidad de Humanidades y Ciencias Sociales, seguido del 17,8 \% que ha realizado Ciencias de la Naturaleza y de la Salud y el resto, un $15,8 \%$ se distribuye entre Artes, Tecnología y otras; en cuanto las alumnas, hay más mujeres en comparación con el porcentaje de hombres que proceden de Ciencias Naturales y de la Salud, siendo este de un 24,3\%; en el caso de la especialidad de Humanidades y Ciencias Sociales representan un $63,5 \%$ y el $12,2 \%$ restante proceden de la especialidad de Artes, Tecnología y otras. (Tabla 2 )

Tabla 2. Distribución de frecuencias según Sexo y Especialidad en bachillerato.

\begin{tabular}{lcccc}
\hline & Hombre & \multicolumn{2}{c}{ Mujer } \\
\hline $\begin{array}{l}\text { ¿Qué especialidad elegiste } \\
\text { en el bachillerato? }\end{array}$ & Frecuencia & Porcentaje válido & Frecuencia & Porcentaje válido \\
\hline Artes & 2 & & & \\
Humanidades y CC Sociales & 29 & 6,4 & 7 & 3,9 \\
Ciencias de la Naturaleza y de & 8 & 17,8 & 115 & 63,5 \\
la Salud & 3 & 6,7 & 5 & 24,3 \\
Tecnología & 3 & 6,7 & 10 & 2,8 \\
Otras & 45 & 100,0 & 181 & 5,5 \\
\hline Total & & & & 100,0 \\
\hline
\end{tabular}

Además de la especialidad, para elaborar el perfil académico se ha preguntado al conjunto de estudiantes sobre la calificación media obtenida en la asignatura de Inglés, tanto en Bachillerato como en la prueba de acceso a la Universidad. Dicha información se considera relevante para conocer el nivel inicial del cual se tiene que partir, para orientar la práctica docente en la materia de Inglés en el Grado de Educación Infantil y en el Grado de Educación Primaria.

En este sentido, como se aprecia en la Tabla 3, tanto mujeres como hombres, $63 \%$ y $62,2 \%$ respectivamente, en Bachillerato han obtenido una nota media en Inglés de aprobado 5-6, seguido de notable 7-8, siendo el porcentaje de hombres ligeramente superior $31,1 \%$ frente al 27,6\% de las mujeres; y la nota media de sobresaliente 9-10, siendo el porcentaje de mujeres más elevado con un $9,4 \%$ que el de los hombres con un $6,7 \%$. 
Tabla 3. Distribución de frecuencias según Sexo y Nota media en bachillerato

\begin{tabular}{|c|c|c|c|c|}
\hline & \multicolumn{2}{|c|}{ Hombre } & \multicolumn{2}{|c|}{ Mujer } \\
\hline $\begin{array}{l}\text { En Bachillerato/FP ¿Qué calificación } \\
\text { media obtuviste en Inglés? }\end{array}$ & $\begin{array}{l}\text { Frecuenc } \\
\text { ia }\end{array}$ & $\begin{array}{l}\text { Porcentaje } \\
\text { válido }\end{array}$ & Frecuencia & $\begin{array}{l}\text { Porcentaje } \\
\text { válido }\end{array}$ \\
\hline Aprobado 5-6 & 28 & 62,2 & 114 & 63,0 \\
\hline Notable 7-8 & 14 & 31,1 & 50 & 27,6 \\
\hline Sobresaliente 9 - 10 & 3 & 6,7 & 17 & 9,4 \\
\hline Total & 45 & 100,0 & 181 & 100,0 \\
\hline
\end{tabular}

En cuanto a la nota media obtenida en el examen de acceso a la Universidad (Tabla 4), también se concentra el mayor porcentaje de mujeres con un $68 \%$ y de hombres con un 71,1 $\%$ en la nota media de aprobado $5-6$, seguido del notable $7-8$ con un $25,4 \%$ de mujeres y un $24,4 \%$ en el caso de los hombres. En lo que respecta al valor de sobresaliente 9-10, al igual que sucede con el notable, se registra un porcentaje mayor de mujeres $(6,6 \%)$ que de hombres $(4,4 \%)$.

Tabla 4. Distribución de frecuencias según Sexo y Nota media en prueba de acceso a la universidad

\begin{tabular}{lcccc}
\hline & & Hombre & & Mujer \\
\hline $\begin{array}{l}\text { Y en Selectividad ¿Qué } \\
\text { calificación obtuviste en Inglés? }\end{array}$ & Frecuencia & Porcentaje válido & Frecuencia & Porcentaje válido \\
\hline Aprobado 5-6 & 32 & & & \\
Notable 7-8 & 11 & 24,4 & 123 & 68,0 \\
Sobresaliente 9 - 10 & 2 & 4,4 & 46 & 25,4 \\
\hline Total & 45 & 100,0 & 12 & 6,6 \\
\hline
\end{tabular}

Otra variable relacionada con el perfil académico que ha sido considerada es "motivos de elección del Grado de Educación", ya que se considera útil a la hora de diseñar el enfoque docente. Partiendo de este criterio, se presenta la Tabla 5, en la que se observa que el mayor porcentaje, tanto en el caso de hombres como en el de mujeres, ha elegido la carrera como primera opción que es lo que siempre han querido estudiar. No obstante, cabe matizar que en el caso de los hombres el porcentaje es de $48,9 \%$ mucho más reducido que el porcentaje de mujeres que registra un $73,5 \%$. En cuanto a los motivos a los que hacen referencia, cabe destacar especialmente la opción "para presentarme a unas oposiciones y esta carrera puedo acabarla rápidamente" donde se han registrado casos en el grupo de los hombres $(6,7 \%)$ pero no en el grupo de las mujeres.

Tabla 5. Distribución de frecuencias según Sexo y motivos de elección del Grado de Educación.

\begin{tabular}{|c|c|c|c|c|}
\hline & \multicolumn{2}{|c|}{ Hombre } & \multicolumn{2}{|c|}{ Mujer } \\
\hline Elegí esta carrera... & Frecuencia & $\begin{array}{l}\text { Porcentaje } \\
\text { válido }\end{array}$ & Frecuencia & $\begin{array}{l}\text { Porcentaje } \\
\text { válido }\end{array}$ \\
\hline $\begin{array}{l}\text {... como primera opción, es lo que siempre he } \\
\text { querido estudiar. }\end{array}$ & 22 & 48,9 & 133 & 73,5 \\
\hline $\begin{array}{l}\text {... porque me gusta, pero no fue la primera } \\
\text { opción. }\end{array}$ & 16 & 35,6 & 43 & 23,8 \\
\hline $\begin{array}{l}\text {... porque no tenía nota para otra carrera que } \\
\text { me hubiera gustado hacer. }\end{array}$ & 4 & 8,9 & 5 & 2,8 \\
\hline $\begin{array}{l}\text { oporque quiero prepararme unas } \\
\text { oposiciones y esta carrera puedo acabarla } \\
\text { rápidamente. }\end{array}$ & 3 & 6,7 & 0 & 0,0 \\
\hline Total & 45 & 100,0 & 181 & 100,0 \\
\hline
\end{tabular}




\section{Las creencias presentes en el aprendizaje del Inglés según el sexo}

Para analizar el conjunto de creencias presentes al comienzo de la asignatura de Inglés en los Grados de Educación Infantil y de Primaria, en primer lugar se realizó un análisis descriptivo según el sexo, considerando las valoraciones medias de los ítems recogidos en la escala Likert (Figura 3).

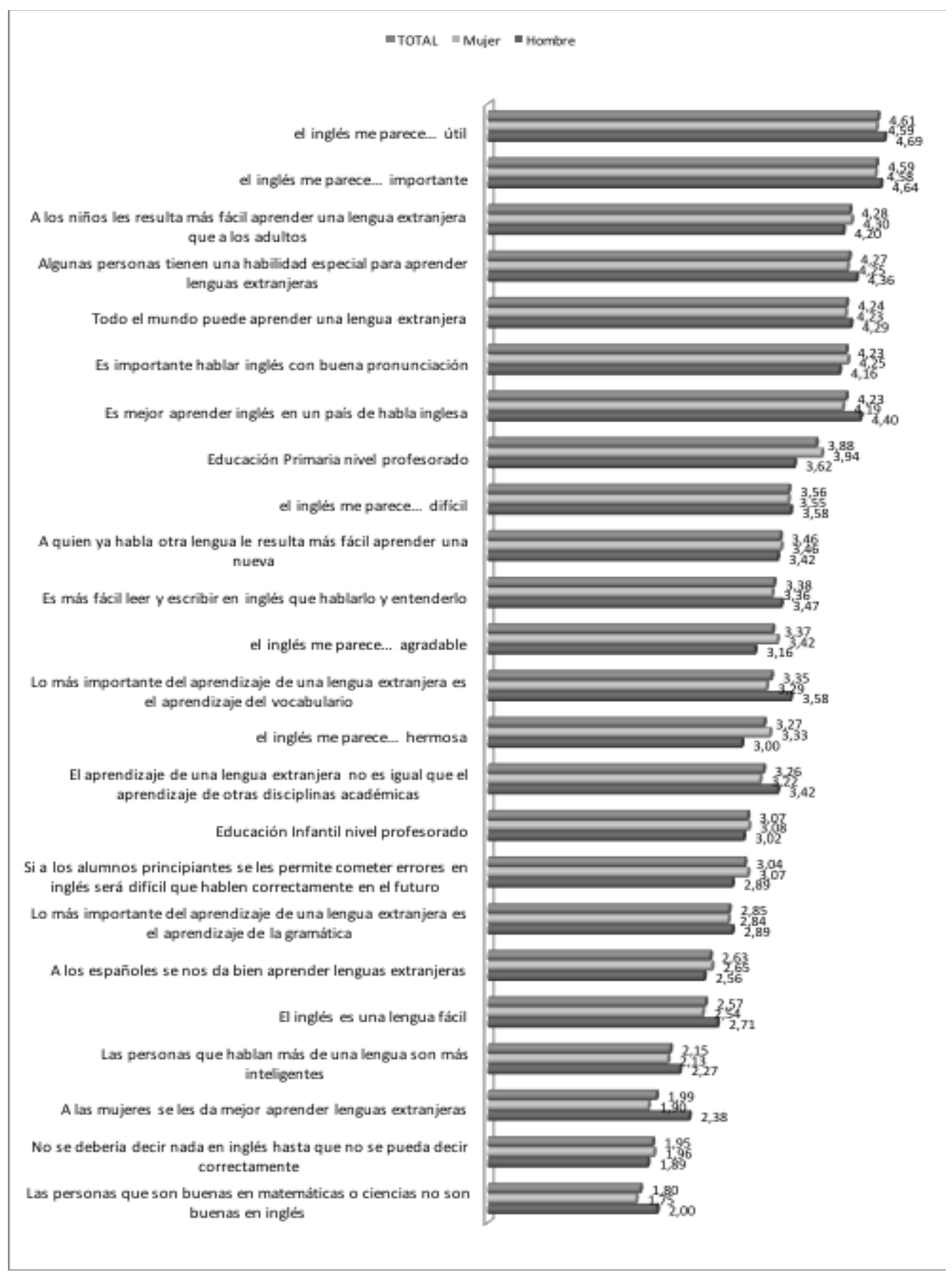

Figura 3. Valores medios de las creencias presentes en el aprendizaje del Inglés según el sexo

Como se pueden observar, los ítems con las que están totalmente en desacuerdo y en desacuerdo y se sitúan en una media por debajo de 3 (ni de acuerdo, ni desacuerdo) son: 
- Las personas que son buenas en matemáticas o ciencias no son buenas en inglés.

- No se debería decir nada en inglés hasta que no se pueda decir correctamente.

- A las mujeres se les da mejor aprender lenguas extranjeras.

- Las personas que hablan más de una lengua son más inteligentes.

- El inglés es una lengua fácil.

- A los españoles se nos da bien aprender lenguas extranjeras.

- Lo más importante del aprendizaje de una lengua extranjera es el aprendizaje de la gramática.

En cuanto a las creencias con las que están de acuerdo y totalmente de acuerdo y se sitúan en una media a partir de 4 (de acuerdo) son:

- Es mejor aprender inglés en un país de habla inglesa.

- Es importante hablar inglés con buena pronunciación.

- Todo el mundo puede aprender una lengua extranjera.

- Algunas personas tienen una habilidad especial para aprender lenguas extranjeras.

- A los niños les resulta más fácil aprender una lengua extranjera que a los adultos.

- El inglés me parece... importante.

- El inglés me parece... útil.

\section{Diferencias en las creencias presentes en el aprendizaje del Inglés según el sexo}

Para conocer las diferencias significativas entre las creencias en torno al aprendizaje del inglés según el sexo del grupo de estudiantes, se realizó en primer lugar la prueba de normalidad de Kolmogorov-Smirnov, y a partir de los resultados obtenidos se realizaron posteriormente pruebas no paramétricas. Concretamente para el análisis de las diferencias según el sexo se utilizó la prueba de $U$ de Mann-Whitney, que como se recoge en la Tabla 6, estas se hallan en el ítem "Lo más importante del aprendizaje de una lengua extranjera es el aprendizaje del vocabulario" ( $p .05)$ y el ítem "A las mujeres se les da mejor aprender lenguas extranjeras" $(p<.01)$.

Tabla 6. Prueba de U de Mann-Whitney. Análisis diferencial de la Escala de creencias presentes en el aprendizaje del Inglés según sexo.

\begin{tabular}{llcc}
\hline & & $\begin{array}{l}U \text { de } \\
\text { Whitney }\end{array}$ & $\begin{array}{c}\text { Sig. } \\
\text { (bilateral) }\end{array}$ \\
\hline $\begin{array}{l}\text { Lo más importante del aprendizaje de una lengua } \\
\text { extranjera es el aprendizaje del vocabulario. }\end{array}$ & 3341,500 &, 047 \\
$\begin{array}{l}\text { A las mujeres se les da mejor aprender lenguas } \\
\text { extranjeras }\end{array}$ & 2932,500 &, 002 \\
\hline
\end{tabular}

Para conocer con mayor precisión las valoraciones de mujeres y hombre, se analizaron los rangos promedios, que como se observa en las Figuras 4 y 5 , son los hombres los que puntúan más en ambos ítems. 


\section{Prueba $U$ de Mann-Whitney de muestras independientes}

Sexo

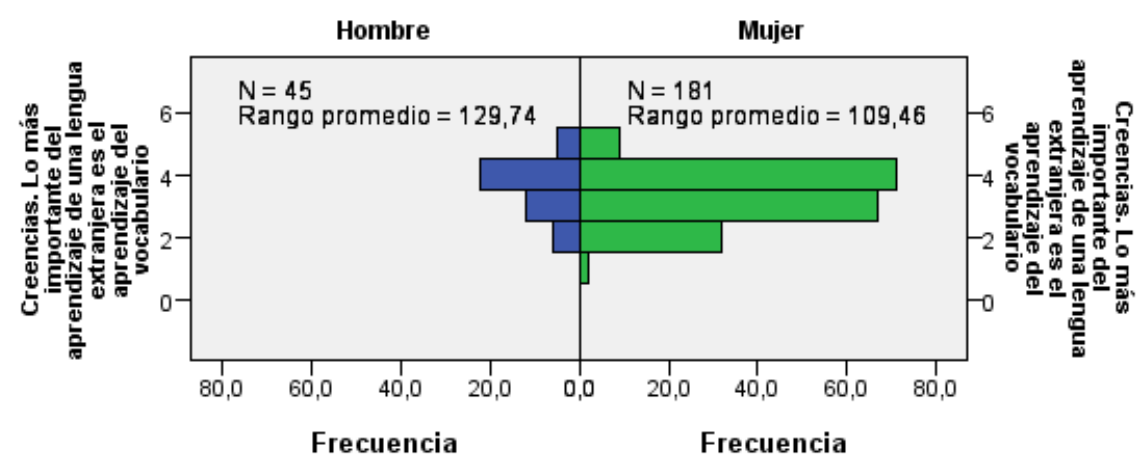

Figura 4. Prueba de U de Mann-Whitney. Rangos según sexo del ítem: "Lo más importante del aprendizaje de una lengua extranjera es el aprendizaje del vocabulario"

Prueba $U$ de Mann-Whitney de muestras independientes

Sexo

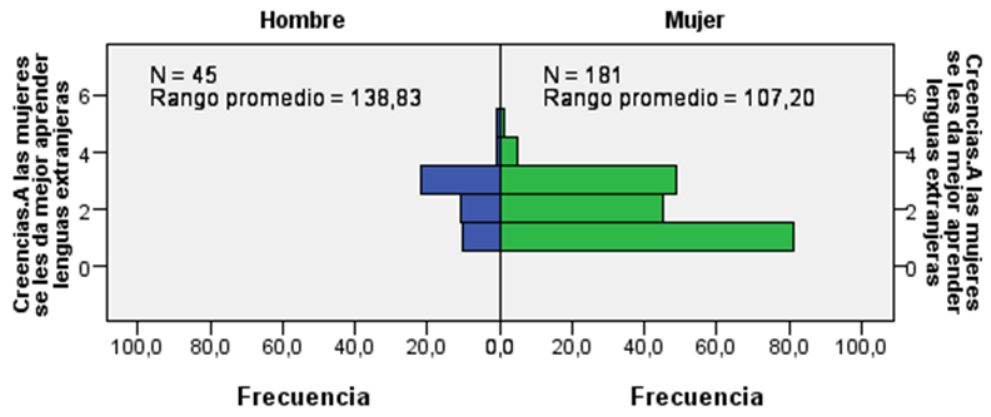

Figura 5. Prueba de U de Mann-Whitney. Rangos según sexo del ítem: "A las mujeres se les da mejor aprender lenguas extranjeras"

\section{Diferencias en las creencias presentes en el aprendizaje del Inglés según el Grado}

En cuanto a las diferencias en las creencias en torno al aprendizaje del inglés según el Grado que cursan actualmente (Grado en Educación Infantil o Grado en Educación Primaria), la prueba de $U$ de Mann-Whitney (Tabla 7$)$ ha recogido diferencias significativas $(p<.05)$ en el ítem "El aprendizaje de una lengua extranjera no es igual que el aprendizaje de otras disciplinas académicas" siendo el grupo de Educación Primaria el grupo que registra un rango promedio mayor a favor de dicha afirmación (Figura 6).

Tabla 7. Prueba de U de Mann-Whitney. Análisis diferencial de la Escala de creencias presentes en el aprendizaje del Inglés según Grado.

$\begin{array}{ll}\text { U de Mann- } & \text { Sig. asintót. } \\ \text { Whitney } & \text { (bilateral) }\end{array}$

El aprendizaje de una lengua extranjera no es igual que el 7.464,500 ,022 aprendizaje de otras disciplinas académicas. 


\section{Prueba U de Mann-Whitney de muestras independientes}

Grado que actualmente cursas

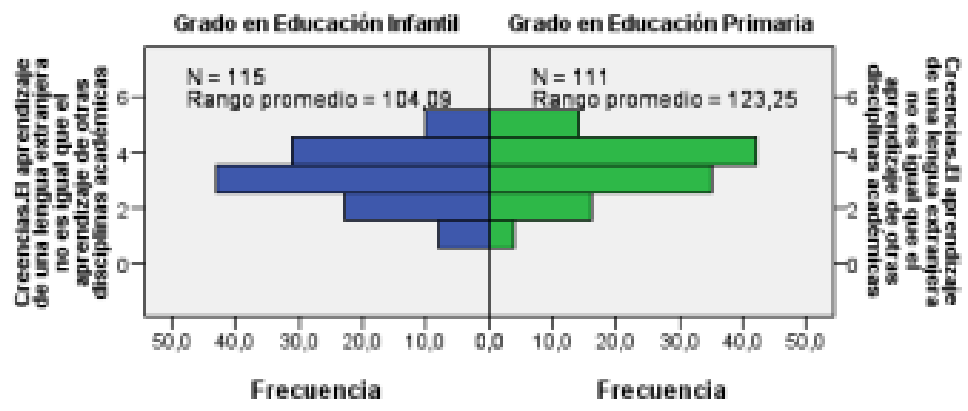

Figura 6. Prueba de U de Mann-Whitney. Rangos según Grado del ítem: "El aprendizaje de una lengua extranjera no es igual que el aprendizaje de otras disciplinas académicas"

\section{CONCLUSIONES}

Para Zimmerman (1990), "las ideas alternativas de los estudiantes se vuelven obstáculos en el aprendizaje, influyendo en sus resultados" (p.12). Por ello es tan importante tener en consideración las creencias presentes en el aprendizaje del inglés. Como señala Barrios (2015), es necesario conocer las creencias preconcebidas, de manera que se puedan adaptar los contenidos de los programas de estudio.

En este sentido, las creencias más compartidas por el grupo de estudiantes y las que tienen un mayor grado de acuerdo son aquellas relativas a que "es mejor aprender inglés en un país de habla inglesa". Esta es una idea bastante extendida entre la población principalmente debido a que se entiende como una manera de aprender mediante la práctica, lo que explica también que valoren positivamente lo importante que es "hablar en inglés con buena pronunciación".

Por otro lado, consideran que "todo el mundo puede aprender una lengua extranjera"; no obstante, expresan que en el aprendizaje del Inglés hay personas que "tienen una habilidad especial para aprender lenguas extranjeras". Se aprecia la creencia de que todo el mundo puede aprender, pero hay personas que tienen más facilidades.

La creencia sobre la idea de la facilidad para aprender Inglés también se atribuye en función de la edad, señalando que a menor edad más facilidad para aprender inglés. De modo que, las creencias vinculadas con características facilitadoras del aprendizaje como la edad o la habilidad de ciertas personas son creencias muy frecuentes entre los estudiantes. De manera que la adquisición de la competencia de la lengua del Inglés no se percibe como un proceso de enseñanza-aprendizaje continuado en el tiempo, sino que parte de este aprendizaje se debe al azar, es decir, a poseer unas habilidades determinadas o porque desde edades tempranas se ha estudiado inglés.

En estas creencias existen elementos facilitadores en el aprendizaje del inglés, pues se han encontrado diferencias en el análisis diferencial según el sexo. En el resultado de este estudio existen diferencias significativas ya que los hombres consideran que "las mujeres se les da mejor aprender lenguas extranjeras", mientras que las mujeres no están nada de acuerdo con esta afirmación. Por tanto, cabe considerar que, entre las creencias ligadas al aprendizaje del inglés, destacan especialmente las creencias relativas a características facilitadoras en el aprendizaje del inglés como es la habilidad, la edad y el género. 
A partir del análisis de los datos obtenidos y de las consideraciones planteadas, y en la línea de las propuestas planteadas por autores como Zimmerman (1990) y Barrios (2014), una alternativa para propiciar que afloren las creencias de modo más espontáneo sería recurrir a estudios cualitativos como entrevistas $u$ otro tipo de instrumentos. En este sentido cabría mencionar aquellos que utilizan metáforas como herramienta para el análisis de las creencias presentes en el aprendizaje (Mellado, Luengo, De la Montaña y Bermejo, 2016). El conocimiento de las creencias y la reflexión sobre la relación entre creencias y prácticas puede ayudar a los maestros a desarrollar una comprensión de lo que hacen en clase y para qué lo hacen, así como de los cambios que pueden aplicar a sus métodos de enseñanza-aprendizaje.

Coincidiendo con Barrios (2014), necesitamos más investigaciones en los centros y facultades de formación de profesorado de inglés y abarcar un contexto geográfico más amplio, para conseguir una mejor información y formación e indagar, más profundamente, sobre cómo las creencias influyen en los contextos de enseñanza y aprendizaje de lenguas extranjeras.

\section{BIBLIOGRAFÍA}

Barrios, M. E. (2006). Dificultades y carencias que experimentan y evidencian futuros docentes de inglés durante su intervención docente autónoma en las prácticas de enseñanza. Revista Electrónica Interuniversitaria de Formación del Profesorado, 9(1), 1-12.

Barrios, M. E. (2015). Evolución de las creencias del profesorado en formación sobre la enseñanza y el aprendizaje del inglés. Bordón 67(2), 45-62.

Barrios, M. E. (2014). Creencias sobre el aprendizaje de una lengua del futuro profesorado de inglés de Educación Primaria. Porta Linguarum, 22, 73-93.

Borg, S. (2003). Teacher cognition in language teaching: a review of research into what language teachers think, know, believe and do. Language Teaching, 36, 81-109.

Borg, S. (2011). The impact of in-service education on language teachers' beliefs. System, 39(3), 370-380.

Cheng, M.M.H., Chan, K.W., Tang, S.Y.F y Cheng, A.Y.N. (2009). Pre-service teacher education students' epistemological beliefs and their conceptions of teaching. Teaching and Teacher Education, 25, 319-327.

Díaz, C. Morales, H. (2015). Creencias de estudiantes de primaria sobre el aprendizaje del inglés en un establecimiento educacional chileno. Revista Electrónica Actualidades Investigativas en Educación 15(1), 1-20.

Espinosa, E. B. (2014). Creencias sobre el aprendizaje de una lengua del futuro profesorado de inglés de Educación Primaria. Porta Linguarum: revista internacional de didáctica de las lenguas extranjeras, (22), 73-93.

Fives, H. y Gill, M.G. (Eds.) (2015) International handbook of research on teachers' beliefs. New York: Routledge.

Horwitz, E. (1999). Cultural and situational influences on foreign language learners' beliefs about language learning: a review of BALLI studies. System, 27, 557-576.

Marín R. (1999). El inglés en la comunicación del futuro. Puertas a la lectura 6(7), 74-79.

Martínez, J.D (2005). Creencias relativas al aprendizaje de una lengua extranjera. Cauce, 28, 219-234.

Martínez, J.D. (2003). Exploración de las creencias de los profesores en formación sobre la enseñanza del inglés como Lengua Extranjera. Campo abierto: Revista de educación, 23, 175-188.

McCarthy, M.J. (1991). Domine la era de la información. Barcelona: Robin Book.

Mellado L., Luengo, M.R., de la Montaña y Bermejo M.L. (2016). Las metáforas emocionales de profesores en formación de infantil, primaria y psicopedagogía. Enseñanza \& Teaching, 34(1), 179195.

Murphy, P., Delli, L. y Edwards, M. (2004). The Good Teacher and Good Teaching: Comparing Beliefs of Second-Grade Students, Preservice Teachers, and Inservice Teachers. Journal of Experimental Education, 72(2), 69-92.

Northcote, M. (2009). Educational beliefs of higher education teachers and students: Implications for teacher education. Australian Journal of Teacher Education (Online), 34(3), 69-81.

Pajares, F. (1992). Teachers' beliefs and educational research: Cleaning up the messy construct. Review of Educational Research, 62(3), 247-265.

Richards, J. C., y Lockhart, C. (1994). Reflective teaching in second language

classrooms. Cambridge: Cambridge University Press.

Talaván, N. (2013). La subtitulación en el aprendizaje de lenguas extranjeras. Barcelona: Octaedro,

Zimmerman, B.J. (1990). Self-regulated learning and academic achievement: An overview. Educational Psychologist, 25(1), 3-17. 